\title{
CINÉTICA DE DEGRADAÇÃO DA COR DA SALSICHA
}

Keeping color of sausage

Cinética de degradación del color de la salsicha

\section{Mariana Silva Araújo*1, Fábio Santos da Silva ${ }^{1}$, Gabriel Montagnini Oliveira $^{1}$, Noé Ferreira Júnior ${ }^{1}$, Victor Hugo Rodrigues ${ }^{1}$, Adriana Régia Marques de Souza ${ }^{2}$}

${ }^{1}$ Discente no Curso de Engenharia de Alimentos, Universidade Federal de Goiás, Goiânia, Brasil.

${ }^{2}$ Doscente do Curso de Engenharia de Alimentos, Universidade Federal de Goiás, Goiânia, Brasil.

*Correspondência: Universidade Federal de Goiás, Avenida Esperança s/n Campus Samambaia, Goiânia, Goiás, Brasil. CEP:74.690-900. E-mail mariiaraujo730@ gmail.com

\section{RESUMO}

A salsicha é um embutido feito a partir da emulsão de carne de diferentes espécies de animais, podendo ser adicionado diversos ingredientes em sua composição. Em produtos cárneos, a sua cor característica é definida pelo conteúdo e a forma da mioglobina. O presente estudo avaliou a variação da cor da salsicha em uma temperatura fixa, variando-se o tempo. Foi utilizado o sistema CIELab para a determinação da cor, sendo avaliado a luminosidade (L) que varia de zero (preto) a 100 (branco), (a*) que varia entre vermelho e verde, (b*) que varia entre amarelo e azul e (c) (croma) que indica a saturação da cor. Com o resultado da cinética da degradação da cor da amostra, observamos uma reação de ordem 0 , sendo então a degradação da cor desta amostra linearmente ligada ao tempo de cozimento.

Palavras-chave: Embutido; Mioglobina; Cor.

\section{ABSTRACT}

The sausage is a stuffing made in the emulsion of meat of different species of animals, as is customary in its composition. In meat products, its color is defined as the content and shape of myoglobin. The present study evaluated a variation of the temperature in a certain measure, varying the time. The CIELab system was used to determine the color, thus a luminosity (L) ranging from zero (black) to 100 (white), ( $\left.a^{*}\right)$ varies between yellow and green, $\left(b^{*}\right)$ varies between yellow and blue and (c) (chroma) indicating a color saturation. With the result of the degradation of the sample of the sample, a solution of order was observed, being a degradation of the sample linearly connected to the cooking time.

Keywords: Built-in; Myoglobin; Color.

\section{RESUMEN}

La salchicha es un embutido hecho a partir de la emulsión de carne de diferentes especies de animales, pudiendo ser agregados diversos ingredientes en su composición. En productos cárnicos, su color característica se define por el contenido y la forma de la mioglobina. El presente estudio evaluó la variación del color de la salchicha a una temperatura fija, variando el tiempo. Se utilizó el sistema CIELab para la determinación del color, siendo evaluado la luminosidad $(L)$ que varía de cero (negro) a 100 (blanco), $\left(a^{*}\right)$ que varía entre rojo y verde, $\left(b^{*}\right)$ que varía entre amarillo y azul y (c) (croma) que indica la saturación del color. Con el resultado de la cinética de la degradación del color de la muestra, observamos una reacción de orden 0 , siendo entonces la degradación del color de esta muestra linealmente ligada al tiempo de cocción.

Descriptores: Incorporada; Mioglobina; Color. 


\section{INTRODUÇÃO}

A instrução normativa $n^{\circ} 4$, anexo IV (Brasil, 2000), define a salsicha como sendo o produto cárneo industrializado, obtido da emulsão de carne de uma ou mais espécies de animais, adicionados de ingredientes, embutido em envoltório natural ou artificial, e submetido a um processo térmico adequado. As salsichas poderão ter como processo alternativo o tingimento, depelação, defumação e a utilização de recheios e molhos (Brasil, 2000).

$\mathrm{Na}$ elaboração dos produtos embutidos emulsionados, além dos produtos cárneos, são adicionados produtos não cárneos, como água, fosfato, sal, gordura (toucinho), proteína vegetal, sais de cura, condimentos, antioxidante e amido, cada um exercendo uma função específica de acordo com sua propriedade (Reis et al., 1999).

A vida de prateleira é um fator de muita importância na indústria de produtos cárneos, pois quanto maior, resulta na diminuição de produtos devolvidos no varejo. Em produtos emulsionados a vida de prateleira é limitada devido à contaminação após cozimento causado pela manipulação, no processo de embalagem e retirada das tripas (Especial carnes, 2017).

A mioglobina é o principal pigmento responsável pela coloração de carnes. Pode existir uma interdependência entre a oxidação lipídica e a alteração dos pigmentos da carne. Para determinação objetiva da cor de alimentos, incluindo carnes e derivados, o sistema CIELab, desenvolvido pela comissão internacional de iluminação. Este sistema avalia a luminosidade (L), variando de zero (preto) a 100 (branco), e os valores de $\left(\mathrm{a}^{*}\right)$ variação entre vermelho e verde, além de ( $\left.b^{*}\right)$ variação entre amarelo e azul. O valor de ( $\mathrm{a}^{*}$ ) é muito importante na análise de produtos cárneos, pois está relacionado com o estado do pigmento cárneo e com a aceitabilidade sensorial (Mancini e Hunt, 2005).
A cinética de degradação da cor em alimentos é complexa. Modelos seguros que predizem corretamente o processo da reação química são proveitosos em muitas aplicações da engenharia incluindo processos de otimização. Estudos experimentais e aplicação de modelos para predizer e interpretar parâmetros cinéticos como a ordem de reação são necessários (Ahmed et al., 2002). Além disso, modelos cinéticos de destruição térmica são essenciais para desenvolver novos processos, assumindo um produto alimentício seguro e fornecendo a máxima retenção dos fatores de qualidade (Ávila e Silva, 1999)

O objetivo deste estudo foi avaliar a cinética da degradação da cor de salsichas em diferentes tempos de cozimento.

\section{MATERIAIS E MÉTODOS}

As salsichas foram adquiridas em supermercado na cidade de Goiânia (GO). Para a avaliação da degradação da cor, as unidades inteiras de salsichas foram cortadas ao meio e dispostas, dentro de recipiente com água (Béquer 1L) aquecido sob chama de bico de Bunsen, a partir do momento em que se atingiu a temperatura aproximada de $90^{\circ} \mathrm{C}$ foi iniciada a contagem. O tempo total foi de 5 minutos procedendo com retiradas, de uma unidade, a cada 30 segundos $(30,60,90,120,150,180,210,240,270$ e 300 segundos). As medidas ao longo do processo foram efetuadas com auxílio de termômetro e cronômetro (Kılıç et al., 2007).

Cada uma das amostras de salsicha submetidas ao cozimento e retiradas no tempo apropriado foram colocadas em pratos plásticos numerados seguindo a ordem cronológica de retirada do cozimento e aguardando até que se atingissem temperatura ambiente para prosseguir com análise em colorímetro.

\section{Análise de cor}


Foi empregado colorímetro modelo ColorQuest II, operando no sistema CIELab, em que $L^{*}$ corresponde a luminosidade, $a^{*}$ e $b^{*}$ são as coordenadas de cromaticidade $(-a=$ verde e $+a=$ vermelho; $-b=$ azul e $+b=$ amarelo) (Harder, 2005). O parâmetro C (Croma) foi calculado de acordo com a Equação 1.

$$
c=a^{2}+b^{2}
$$

(Equação 1)

\section{Parâmetros cinéticos}

Os dados de cor obtidos pelos parâmetros $\mathrm{a}^{*} \mathrm{e}$ $\mathrm{b}^{*}$, para cada tempo, foram analisados quanto à ordem de reação de alteração da cor. A ordem de reação (n) da alteração da cor das salsichas, a constante da velocidade de reação $(\mathrm{k})$ e o tempo de meia vida $(\mathrm{t}$ 1/2) (Labuza, 1982).

A cinética dos dados experimentais foi apresentada na forma adimensional $\mathrm{C} / \mathrm{C}_{0}$ em diferentes intervalos de tempo de aquecimento ( $\mathrm{t}$ ), onde $\mathrm{C}$ é a cor resultante da leitura das coordenadas $\left(L^{*}, a^{*}\right.$ e $\left.b^{*}\right)$ depois de cada tratamento e $\mathrm{C}_{0}$ é a cor $\left(\mathrm{L}^{*}, \mathrm{a}^{*}\right.$ e $\left.\mathrm{b}^{*}\right)$ da amostra obtida no tempo 0 (Achir et al., 2010). A taxa de degradação inicial (r) de cada parâmetro avaliado foi encontrada através da medida da inclinação da tangente da curva $\mathrm{C} / \mathrm{C}_{0}=\mathrm{f}(\mathrm{t})$ para $\mathrm{t}=0$.

A ordem de reação (n) foi determinada por meio da plotagem dos valores das coordenadas de cor versus o tempo de aquecimento em cada tratamento. Foi realizada a correlação linear destes pontos e o melhor ajuste foi selecionado usando como critério o coeficiente de determinação (R2). A constante da velocidade de reação $(k)$ foi determinada por meio do coeficiente angular da reta que determinou a ordem da reação.

O tempo de meia vida $\left(\mathrm{t} \frac{1 / 2}{2}\right)$ foi determinado por meio do tempo necessário para que os valores das coordenadas de cor se reduzissem à metade do valor inicial, utilizando umas das seguintes equações, conforme a ordem de reação determinada: ordem zero $\mathrm{t} 1 / 2=[\mathrm{C}] / 2 \mathrm{k}$; primeira ordem $\mathrm{t} 1 / 2=\ln 2 / \mathrm{k}$; segunda ordem $\mathrm{t} 1 / 2=1 / \mathrm{k}\left[\mathrm{C}_{0}\right]$.

\section{RESULTADOS E DISCUSSÃO}

\section{Parâmetros cinéticos}

A diminuição do parâmetro Croma indicou o escurecimento das amostras, o que indica uma cor menos saturada (Figura 1).

Rodrigues et al. (2013), constatou que a degradação da cor de azeite de pequi foi de ordem zero, indicando que as alterações que ocorrem na coloração do mesmo dependem do tempo de exposição à temperatura empregada. Estudos de Ferreira et al. (1999) avaliou a degradação de cor com a variação de temperatura em solução de urucum e verificou que as alterações de cor são dependentes da temperatura e do tempo de tratamento. As reações de degradação de cor e os teores de pigmentos foram moderadamente afetados pela temperatura, praticamente dobrando sua velocidade a cada $10{ }^{\circ} \mathrm{C}$ de aumento.

Figura 1. Cinética de ordem 0 (A), 1 (B) e 2 (C) da degradação de cor de salsicha em diferentes tempos de cozimento.
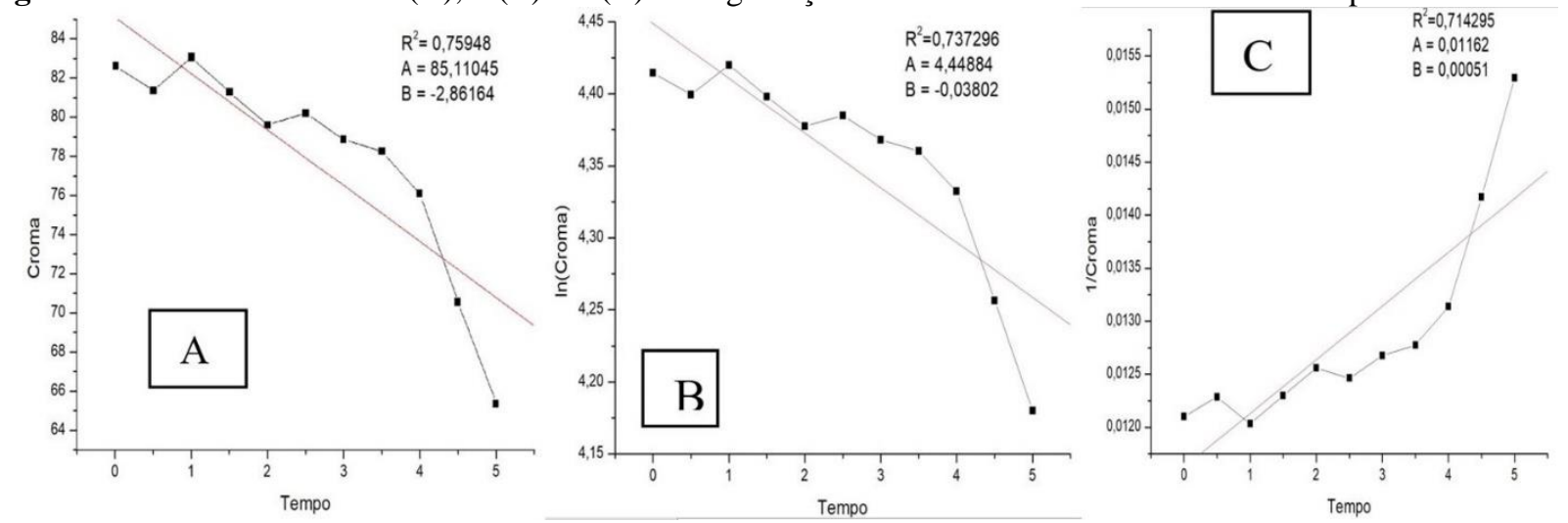
De acordo com os resultados cinéticos (Tabela 1), foi observado que a cinética de degradação da cor de salsicha, para todos os parâmetros avaliados $\left(\mathrm{L}, \mathrm{a}^{*}\right.$ e $b^{*}$ ), durante seu cozimento foi de ordem 0 , obtendo maior regressão linear $\left(\mathrm{R}^{2}\right)$. Isto indica que a reação de degradação da cor da amostra acontece de forma linear ao tempo, com uma proporção de 1:1, conforme aumenta o tempo de cozimento, linearmente, aumentase a degradação da cor da salsicha.

Tabela 1. Parâmetros cinéticos da alteração de cor de salsichas ao longo do tempo de aquecimento.

\begin{tabular}{cccccc}
\hline Parâmetro & $\mathbf{n}$ & Regressão linear & Coeficiente de determinação & $\mathbf{k}$ & $\mathbf{t}_{1 / 2}$ \\
\hline \multirow{2}{*}{ Cor $\mathrm{L}^{*}$} & 0 & $\mathrm{y}=-2,3 \mathrm{x}+45,504$ & $\mathrm{R}^{2}=0,8146$ & 0,13 & 168,73 \\
& 1 & $\mathrm{y}=-0,0612 \mathrm{x}+3,8294$ & $\mathrm{R}^{2}=0,7698$ & 0,18 & 3,85 \\
& 2 & $\mathrm{y}=0,0016 \mathrm{x}+0,0214$ & $\mathrm{R}^{2}=0,7698$ & 0,29 & 0,07 \\
\hline \multirow{2}{*}{ Cor $\mathrm{a}^{*}$} & 0 & $\mathrm{y}=0,8471 \mathrm{x}+35,774$ & $\mathrm{R}^{2}=0,5016$ & 0,22 & 40,65 \\
& 1 & $\mathrm{y}=-0,0228 \mathrm{x}+3,5765$ & $\mathrm{R}^{2}=0,4975$ & 0,20 & 3,46 \\
& 2 & $\mathrm{y}=0,0006 \mathrm{x}+0,028$ & $\mathrm{R}^{2}=0,4925$ & 0,17 & 0,16 \\
\hline \multirow{2}{*}{ Cor $\mathrm{b}^{*}$} & 1 & $\mathrm{y}=-0,059 \mathrm{x}+4,3611$ & $\mathrm{R}^{2}=0,8011$ & 0,14 & 66,53 \\
& 2 & $\mathrm{y}=0,0009 \mathrm{x}+0,0126$ & $\mathrm{R}^{2}=0,7575$ & 0,18 & 3,85 \\
& & & $\mathrm{R}^{2}=0,7117$ & 0,29 & 0,046
\end{tabular}

Constantes de velocidade de reação $(\mathrm{k})$; tempo de meia vida (t 1 1 2 )

O tempo de meia vida, expresso em minutos, apresenta o decréscimo dos valores de $\mathrm{a}^{*}$ (vermelho) até 80 minutos de aquecimento, ou seja, o tempo mínimo para que a metade das reações comecem a acontecer, enquanto o aumento dos valores de $b^{*}$ (amarelo) se inicia aos 40 minutos, apresentando menor tempo para a reação iniciar, ou seja, degradando para coloração amarelo mais rapidamente (Tabela 1).

Quanto menor o tempo de meia vida, maior a velocidade de degradação da reação, neste caso, podese constatar que quanto mais elevada a temperatura, menor será o tempo de meia-vida, consequentemente maior degradação da coloração das salsichas. A velocidade de reação $(k)$ interfere diretamente no tempo de meia vida da reação, sendo esta constante influência pela variação da temperatura.

Bastos et al. (2015) constataram em seu estudo que as betaninas degradam mais rapidamente às temperaturas mais altas, já que o tempo de meia-vida, ou seja, tempo em que a concentração inicial reduz à metade, diminuiu ao passo que a temperatura de trabalho foi aumentada.

\section{CONCLUSÃO}

A reação de degradação da cor da salsicha em relação ao tempo de cozimento é de ordem 0 , ou seja, o escurecimento da salsicha está linearmente ligado ao tempo de cozimento da mesma.

Todos os autores declararam não haver qualquer potencial conflito de interesses referente a este artigo.

\section{REFERÊNCIAS}

ACHIR, N.; RANDRIANATOANDRO, V. A.; BOHUON, P.; LAFFARGUE, A.; AVALLONE, S. Kinetic study of $\beta$-carotene and lutein degradation in oils during heat treatment. European Journal of Lipid Science and Technology, Weinheim, v.112, n.3, p.349- 361, 2010.

AHMED, J.; SHIVHARE, U. S.; SANDHU, S. Thermal degradation kinetics of carotenoids and visual color of papaya puree. J. Food Sci., v. 67, p. 26922695, 2002. 
ÁVILA, I. M. L. B.; SILVA, C. L. M. Modelling kinetics of thermal degradation of colour in peach puree. J. Food Eng., v. 39, p. 161-166, 1999.

BASTOS, B. M.; GRANDINI, C. P.; RODRIGUES, S. K. C.; SANTOS, M. A.; LOPES, T. S.; ANTELO, F. S. Avaliação da cinética de degradação térmica de betalaínas extraídas de Beta vulgaris L. Blucher Chemical Engineering Proceedings, v. 1, n. 2, p. 3916-3923, 2015.

\section{BRASIL. Ministério da Agricultura e do} Abastecimento. 2000. Disponível em: http://www.adapar.pr.gov.br/arquivos/File/GTA/Legi slacao/Legislacoes_Manual_Fiscalizacao_Transito_A gropecuario/IN_Mapa_04_2000.pdf. Acessado em: 18/11/2017.

ESPECIAL CARNES. 2017. Aditivos \& Ingredientes na Industria da Carne. Disponível em: http://aditivosingredientes.com.br/upload_arquivos/2 01602/2016020202008001454333346.pdf. Acessado em: 18/11/2017.

FERREIRA, V.L.P; NETO; R.O.T; MOURA, S.C.S.R; SILVA; M.S. Cinética da degradação da cor de solução hidrossolúvel comercial de urucum, submetida a tratamentos térmicos. Ciência e Tecnologia de Alimentos, v. 19, n. 1, p. 37-42, 1999.

HARDER, M. N. C. Efeito do urucum (Bixa orellana) na alteração de características de ovos de galinhas poedeiras. 2005. 74 p. Dissertação (Mestrado) Universidade de São Paulo, Piracicaba, 2005.

KILIÇ, K.; ONAL-ULUSOY, B.; BOYACI, İ. H. A novel method for color determination of edible oils in $L^{*} a^{*} b^{*}$ format. European Journal of Lipid Science and Technology, v. 109, n. 2, p. 157-164, 2007.

LABUZA, T.P. Shelf-life dating of foods. Food \& Nutrition Press, Westport, v. 81, n. 1, p. 153, 1982.

MANCINI, R. A.; HUNTI, M. C. Current research in meat color. Meat science, v. 71, n. 1, p. 100-121, 2005.

REIS, R. A. A.; SANTOS, W. L. M.; OLIVEIRA, A. L.; SOUZA, R. M.; VELOSO, C. R. V. Quantificação da hidroxiprolina como índice de qualidade de salsicha comercializada em Belo Horizonte-MG Quantification of hydroxyproline as an index of quality for frankfurter sold in Belo Horizonte, Brazil. Arquivo Brasileiro de Medicina Veterinária e Zootecnia, v. 51, n. 6, p. 589594, 1999.

RODRIGUES, M.L; SOUZA, A.R.M; LIMA, J.C.R; MOURA, C.J; GERALDINE, R.M; TAKEUCH, K.P. Cinética de alteração da cor do azeite de pequi (Caryocar brasiliense Camb.) Submetido a Tratamento Térmico. Cienc. Rural vol.43 no.8 Santa Maria Aug. 2013. 